Mónica Ahumada Figueroa*

\title{
Chile y China: una mirada retrospectiva a 50 años de relaciones diplomáticas y de amistad
}

\section{Chile and China: A Retrospective View to Fifty Years of Diplomatic Relations and Friendship}

https://doi.org/10.1515/sai-2021-2007

Publicado en línea agosto 25, 2021

Resumen: Las relaciones diplomáticas entre Chile y China, han cumplido cinco décadas y estas se caracterizan por los fuertes lazos construidos en forma bilateral, como también con otros países de América Latina y el Caribe. El presente artículo busca indagar sobre esta trayectoria desde los contactos de los años 50 con José Venturelli, un pionero, junto a otros viajeros en el marco de una diplomacia cultural que facilitó la firma de relaciones diplomáticas en 1970. Sin embargo, la reunión APEC celebrada en Santiago de Chile en el 2004 fue el punto de inflexión con la visita del presidente $\mathrm{Hu}$ Jintao impulsándose una estrategia comercial. Así se pasó desde los vínculos políticos y culturales, a una relación marcada por acuerdos económicos y la firma de un Tratado de Libre Comercio. Este nuevo escenario facilitó un estilo de cooperación abierta hacia el ámbito tecnológico y científico, pero también hacia un fortalecimiento de los acuerdos ya firmados. Esto nos permite hacer hoy un análisis desde las variables políticas, económicas, culturales y científicas buscando responder a la pregunta sobre los aspectos que han sido beneficiosos como también los puntos más débiles al querer ambos estados proyectarse dentro del sistema internacional.

Palabras claves: diplomacia cultural, APEC, Tratado de Libre Comercio, intercambio comercial Chile-China, convenios tecnológico-científicos

\footnotetext{
*Corresponding author: Mónica Ahumada Figueroa, Dra. en Estudios Americanos-Relaciones Internacionales, académica del Departamento de Literatura y Lingüística aplicada a la traducción, Facultad de Humanidades, Universidad de Santiago de Chile, Santiago de Chile, Chile, https:// linguisticayliteratura.usach.cl/, E-mail: monica.ahumada@usach.cl
} 
Abstract: Diplomatic relations between Chile and China have lasted for five decades and are characterized by the strong ties built bilaterally as well as with other Latin American and Caribbean countries. This article looks upon this trajectory from the contacts in the 1950s with José Venturelli, a pioneer, along with other travelers within the framework of a cultural diplomacy that eased the signature of diplomatic relations in 1970. However, the APEC Summit celebrated in Santiago, Chile in 2004 was the turning point with the visit of President $\mathrm{Hu}$ Jintao promoting a commercial strategy. Thus, we changed from the political and cultural connections to a relationship marked by economic agreements and the signature of a Free Trade Agreement. This new scenario eased an open cooperation style towards the technological and scientific field, but also towards a strengthening of the already signed agreements. This allows us today to analyze the politic, economic, cultural, and scientific variables seeking to respond the question over the beneficial aspects as well as the weaker points when both states want to project within the international system.

Keywords: cultural diplomacy, APEC, Free Trade Agreement, Chile-China commercial exchange, technologic-scientific agreements

\section{Introducción}

Transcurridos ya 50 años de la firma de relaciones diplomáticas y 15 años de la firma del Tratado de Libre Comercio (TLC) entre Chile y China, surge la necesidad de dar una mirada por un lado a las relaciones ininterrumpidas que han mantenido ambos Estados, al igual que analizar los vínculos de amistad y de reciprocidad que se han ido acrecentando de manera sorprendente desde la firma del TLC. Aspectos esenciales dentro de esta mirada común han sido reconocerse en las profundas asimetrías entre ambos Estados, como también valorar el quehacer mutuo fortaleciéndose el desarrollo político, económico, cultural y científico hasta llegar al día de hoy. El valor de la confianza mutua, ha permitido construir puentes que indiscutiblemente han sido la clave para poder avanzar con un proyecto común de desarrollo.

China es hoy la segunda potencia mundial, con un liderazgo indiscutible del Presidente Xi Jinping a nivel mundial y con un respaldo unánime a su política luego del XIX Congreso del Partido Comunista Chino (Spanish.xinhuanet, 2017), abriéndose un espacio privilegiado frente a Estados Unidos, Rusia y la Unión Europea. Esto teniendo presente que uno de los principales logros fue haber sacado al país de la pobreza y mantener una tasa de crecimiento sostenido por décadas que se expresó en tasas sobre el 10\%. Su índice de crecimiento fue de 6,1 \% en el 2019, siendo un referente frente a cualquier economía (Banco Mundial, 2021a, 
2021b). A su vez, Chile, con una población de 19116210 millones (Banco Mundial, 2021c), consiguió una inserción positiva en el sistema mundial, al lograr desde el regreso a la democracia en los años 90 elevar sus índices de productividad con tasas de crecimiento que bordearon en forma constante sobre el 5\%. Esto permitió que un alto porcentaje de la población pudiera tener acceso a niveles de vida mejor, expresándose en un aumento del poder adquisitivo y un mayor acceso a la educación, a la salud y a la vivienda en forma comparativa. Con respecto al ingreso percápita de Chile, según la Pariedad del Poder Adquisitivo en el periódo señalado 1990-2017, este fluctuó entre los US\$5 839 y los US\$24 588 respectivamente (Economía y Negocios, 2017). Sin embargo, estos datos están muy lejos de la realidad actual del país. Al considerar estadísticas para el año 2019, Chile tuvo un crecimiento de 1,1\% (Banco Mundial, 2021a, 2021b). Esto fue producto de una severa constricción de su economía, siendo una variable determinante la baja en el precio del cobre a nivel mundial y la crisis económica interna vivida, producto de las fuertes demandas sociales y la crisis del empleo.

Conociendo estos antecedentes y estadísticas, se debe destacar el desarrollo actual de ambos países, observando sus políticas de Estado donde el énfasis se ha puesto en elevar las condiciones de vida de su población buscando nuevas oportunidades. Desde esta perspectiva, Chile y China han recorrido un camino con una mirada común que hoy se abre en diferentes áreas. Los acuerdos de cooperación recientes en el ámbito científico, producto del coronavirus, permitieron un proyecto conjunto con la Universidad Católica de Chile en la búsqueda y factibilidad de una vacuna Sinovac para posteriormente ser implementada con una vacunación masiva de la población chilena. Junto a este aspecto tan reciente y determinante en el contexto mundial, es necesario hacer referencia a las recientes inversiones chinas en Chile el año 2020, en materia de energía eléctrica, con la firma de un acuerdo entre State Grid-SGID y la Empresa CGE, principal distribuidora de energía eléctrica en Chile, comprando las acciones de dicha empresa como se verá más adelante (El Pulso, 2020).

Sin embargo, en ningún momento podemos olvidar que los primeros vínculos en los años 50 partieron desde lo cultural, con la figura pionera de José Venturelli y desde lo político, con la cercanía ideológica al líder del socialismo chileno Salvador Allende, acrecentándose durante su gobierno del que él era Presidente de Chile, con la firma de relaciones diplomáticas en 1970 (MINREL,1970).

Posteriormente a fines de los años 80, ambos Estados se abrieron a una complementación de sus economías con una balanza comercial creciente que condujo finalmente a la firma de un TLC en el 2005. De alguna manera, este artículo busca explorar y analizar esta relación bilateral donde las variables a considerar, como ya se dijo, serán las políticas, económicas, culturales y cientifícas, siendo un punto medular, una mirada retrospectiva a la firma del TLC y los últimos acuerdos firmados en un contexto de pandemia mundial. 
Los logros obtenidos entre Chile y China son producto de una relación de amistad y de un trabajo que se sustenta en la confianza mutua, permitiendo nuevos espacios extraregionales en donde a Chile le favoreció insertarse en China para posteriormente ingresar a otros mercados asiáticos. En el caso del gigante asiático, pudo estrechar no sólo lazos con Chile, sino también con América Latina y el Caribe, logrando así aliados estratégicos que le facilitan conseguir un escenario propio del siglo XXI (Wu, 2011, p. 77).

\section{Relaciones diplomáticas entre Chile y China desde sus hitos pioneros}

Las relaciones entre Chile y China, se construyeron a partir de la década del 50 cuando actores no estatales chilenos fueron invitados a visitar la recién proclamada República Popular China. Esto implicó que en forma pionera en 1952, José Venturelli, pintor y muralista chileno, fijara su residencia en el país asiático y estableciera un estrecho vínculo con el Presidente Mao Zedong al nombrarlo Embajador Latinoamericano y organizador del Congreso Mundial por la Paz, de Asia, África y el Pacífico (Mancilla, 2003, p. 66). Esto le permitió establecer una estrecha amistad junto a su familia, con el Primer Ministro Zhou Enlai que se mantendría por más de dos décadas.

Al liderazgo del pintor chileno, se sumaron otras visitas como las figuras de Salvador Allende en 1954, vicepresidente del Senado chileno, quien llegó a Beijing en una visita de amistad y recorrió el país navegando por el río Yangtze en un viaje que se prolongó por más de un mes en una visita privada. Luego, en el contexto de los Congresos Internacionales por la Paz, Pablo Neruda, escritor chileno, fue el primer poeta chileno en ser traducido al chino visitando la RPCH en los años $1951 \mathrm{y}$ 1957 (Neruda, 1992, p. 327). Estos acontecimientos, sumados a otras visitas extendidas por el Instituto chileno-chino de cultura (ICCC), fundado en 1952 dada la gestión de Neruda, Venturelli y Allende, le permitieron a Chile un espacio privilegiado. Al regreso, estas delegaciones nacionales podían difundir la nueva China como también dar a conocer la ideología del movimiento maoísta.

A los antecedentes expuestos, se suman cinco hitos que culminaron con la firma del Tratado de Libre Comercio entre Chile y China, siendo estos un eje articulador en la estrategia asumida por ambos Estados. Ellos son, en orden cronológico: la firma de relaciones diplomáticas el 15 de diciembre de 1970, siendo el primer país de Sudamérica en reconocer la República Popular de China (MINREL, 1970); el apoyo decidido de Chile en la $26^{\mathrm{a}}$ Asamblea General de las Naciones Unidas al exigir "la restitución de los legítimos derechos de la República Popular” y su ingreso a la ONU 
en la Resolución 2758 del 25 octubre de 1971 (Vera Castillo, 1987, p. 310); el compromiso de Chile al impulsar el ingreso a la Organización Mundial de Comercio-OMC de China en diciembre 2001 (Jiang, 2006, p.13); el reconocimiento a China como una economía de mercado dentro del marco de la visita del Presidente $\mathrm{Hu}$ Jintao en el año 2004 con motivo de la APEC (Lagos, 2004) y, por último, al año siguiente, el 2005, la negociación de un TLC Chile-China , siendo el primer país que logró firmar un acuerdo de esta naturaleza fuera de los países ASEAN (SUBREI, 2021).

\section{Chile y China en el contexto diplomático- económico de la firma del TLC}

La firma del TLC entre Chile y China fue el producto de años de un trabajo conjunto en el ámbito diplomático y económico donde el reconocimiento mutuo como economías estables, sólidas, permitió tanto a los actores estatales representantes del Estado chileno, como a los actores no estatales en su calidad de individuos $u$ organizaciones gremiales, visualizar a China como una gran oportunidad y un ejemplo de ello es el sector empresarial chileno. La realización de la APEC ${ }^{1}$ en Chile el año 2004, le permitió a Chile abrir un espacio frente a las 21 economías del AsiaPacífco y al ser país anfitrión pudo explicitar su política exterior de inserción internacional. Al recordarlo, el expresidente Ricardo Lagos, en una entrevista realizada por la autora en 2007, señaló:

El comercio ayuda, pero ayuda mucho más cuando hay también relaciones políticas fluídas. Esto, en la cantidad de visitas en uno u otro sentido y es muy fuerte en ambas direcciones. Dado el tamaño de Chile, el que ud. haya tenido en un período de tres años una visita de Estado del Presidente Jiang Zemin y tres años después de Hu Jintao no es lo habitual para un país como Chile, pero tiene que ver con una cierta percepción china de que ellos pueden desde aquí ampliar su presencia en América Latina (Lagos, 2007).

Esto implicó una fuerte dosis de pragmatismo por parte de ambos Estados, donde los beneficios a largo plazo quedaron demostrados. Las misiones empresariales y las giras de autoridades implicaron en primer lugar conocerse y respetarse en los propios códigos culturales. En segundo lugar, saber cuáles eran los productos que los chinos demandaban y que eran posible satisfacer desde el mercado chileno. En tercer lugar, establecer medidas de confianza que permitieran realizar un trabajo

1 APEC. Asian Pacific Economic Cooperation. Foro multilateral creado en 1989 que permitió agrupar a 21 países pertenecientes a la Cuenca del Pacífico, y que ha permitido desarrollar políticas comunes en materia de integración económica, acuerdos en materia de cooperación técnica y defensa del comercio. Chile se integró como miembro pleno en 1994. 
serio, confiable, continuo y, en cuarto lugar, identificar los productos que eran sensibles para ambos Estados, al momento de discutir las rebajas arancelarias. Así, China y Chile sentaron las bases para futuras negociaciones.

Una década después, al solicitársele una mirada retrospectiva sobre el TLC, el expresidente Ricardo Lagos, en entrevista realizada por la autora en 2015, al respecto señaló:

Nos pareció importante dar el primer paso, era un poco sorpresivo para otros, pero a nosotros nos pareció que debíamos adelantarnos y asegurarnos el acceso a ese mercado. Siempre ser el primero lleva una ventaja y me lo dijo el Primer Ministro de Singapur, ${ }^{2}$ y así nos adelantamos 5 años (...), porque en cinco años más nuestros competidores ${ }^{3}$ van a estar firmando los mismos acuerdos con otros países. Había que asegurar el comercio de bienes, y ya después mirar las inversiones y otros aspectos (Lagos, 2015).

El acuerdo firmado permitió establecer un nuevo marco de cooperación entre ambos Estados fortaleciendo el intercambio comercial, y en una etapa siguiente generar una agenda con proyecciones hacia el sector de servicios, para luego avanzar en materia de inversiones chinas en el país. Estas negociaciones implicaban la compra de materias primas, como el cobre y la celulosa como los productos más importantes, seguidos por productos alimenticios. Así las rebajas arancelarias, al firmarse el acuerdo, favorecerían el comercio entre ambos Estados teniendo un período de desgravación de 10 años. El crecimiento de la balanza comercial fue evidente y desde China, el sector industrial y automotriz entre otros, comenzaron a ocupar un espacio relevante en el mercado nacional, impactando también en un cruce intercultural.

Cabe decir que la política exterior de Chile se enfocó, en esa década, en acuerdos de largo plazo y China fue parte de esta política de Estado, que se expresó en un Regionalismo Abierto ${ }^{4}$ buscando una apertura en el sistema mundial y un espacio

2 Estados Unidos y Singapur, habían firmado en ese momento un TLC con un largo proceso de negociaciones. La intención de iniciar las conversaciones para acordar un TLC, se dio en Washington DC. el 16 de noviembre de 2000. Se realizaron seis rondas de negociaciones y cinco adicionales. Finalmente, este se firmó el 6 de mayo de 2003 y los ámbitos que abarcó eran en materia de comercio de mercancías, y otros.

3 Es interesante señalar que Perú firmó su TLC con China el 2009, y la negociación anterior realizada por Chile les sirvió de modelo, según lo comentó en entrevista de estudio realizada en Lima, 2012, a esta investigadora, el propio embajador de Perú en China en el gobierno de A. Toledo, Luis Chang.

4 Concepto introducido por CEPAL, que busca una estrategia de participación y de inserción de los países en organismos multilaterales. La política de inserción mundial aplicada por Chile desde los años 90 responde a este modelo y facilitó la firma de un gran número de TLC, donde el pragmatismo más allá de las ideologías imperantes fue un sello. Esto le permitió a Chile abrirse un espacio reconocido en el sistema mundial. 
en organismos multilaterales. Desde esta perspectiva, la apertura económica hacia nuevos mercados fue una constante, reflejándose esto en diferentes tipos de negociaciones firmadas en el ámbito económico y comercial.

\section{Escenario económico e intercambio comercial al momento de la firma del TLC en 2005}

En el año 2005, América Latina se caracterizaba por una marcada tendencia hacia la exportación de comodities reflejándose ese año en un crecimiento económico de la región de un 4,3 \% y en el caso de Chile era de un 5,9 \% (DIRECON, 2006, p. 22) siendo esto resultado de las exportaciones de cobre mundiales a un precio privilegiado. Los socios comerciales de Chile eran Estados Unidos, cuyo crecimiento anual era de 3,5 \%, ese año (DIRECON, 2006, p. 6). La demanda de productos chilenos en el mercado norteamericano se mantenía constante expresándose en un alto prorcentaje de exportaciones de cobre, celulosa, salmones, frutas y vinos, que en su totalidad correspondían a US\$6 531,6 millones. El otro socio comercial de Chile era la Unión Europea, manteniendo con ella un TLC vigente desde el 2003 , que le permitió aumentar significativamente las exportaciones de productos del mar y frutas, expresándose en US\$9 177,6 millones (DIRECON, 2006, p. 8).

Con respecto al mercado asiático, Chile firmó un TLC con Corea el 2003 en el marco de su política exterior de Regionalismo Abierto, sin embargo, el mayor valor de exportaciones hacia esa región lo ocupaba Japón, expresadas en minerales y productos del mar alcanzando los US\$4 592,1 millones. China era el cuarto socio comercial y el total de las exportaciones eran la cifra de US\$4 445,7 millones (DIRECON, 2006, p. 110). Es muy interesante destacarlo ya que a partir de la entrada en vigencia del TLC con China el $1^{\circ}$ de octubre del 2006, las exportaciones crecieron rápidamente y el intercambio comercial entre ambos Estados aumentó significativamente produciéndose un giro en el comercio exterior chileno. Así este intercambio que en el 2005 era de US\$ 8 122,7 millones, ya al 2010 era de US\$27 295,2 y al 2019 de US39 126,1 millones reflejando el impacto que este acuerdo comercial produjo.

A partir de las estadísticas revisadas y según los datos proporcionados por la DIRECON y su Departamento de Estudios para el período 2006-2013 y los entregados por la SUBREI, Subsecretaría de Relaciones Económicas Internacionales, para el período 2014-2019, el intercambio comercial puede ser visualizado a continuación en el siguiente gráfico. Cabe decir que la SUBREI es la sucesora de la Dirección General de Relaciones Económicas Internacionales (DIRECON) desde el 1 de julio de 2019 (SUBREI, 2019) (Gráfico N¹).

Al observar los datos estadísticos cabe resaltar la curva ascendente a partir de la entrada en vigencia del acuerdo en 2006. Otro punto interesante es la caída en la balanza comercial producto del inicio de la crisis en Estados Unidos en el año 2008. 


\section{INTERCAMBIO BILATERAL CHILE Y CHINA 2006-2019.}

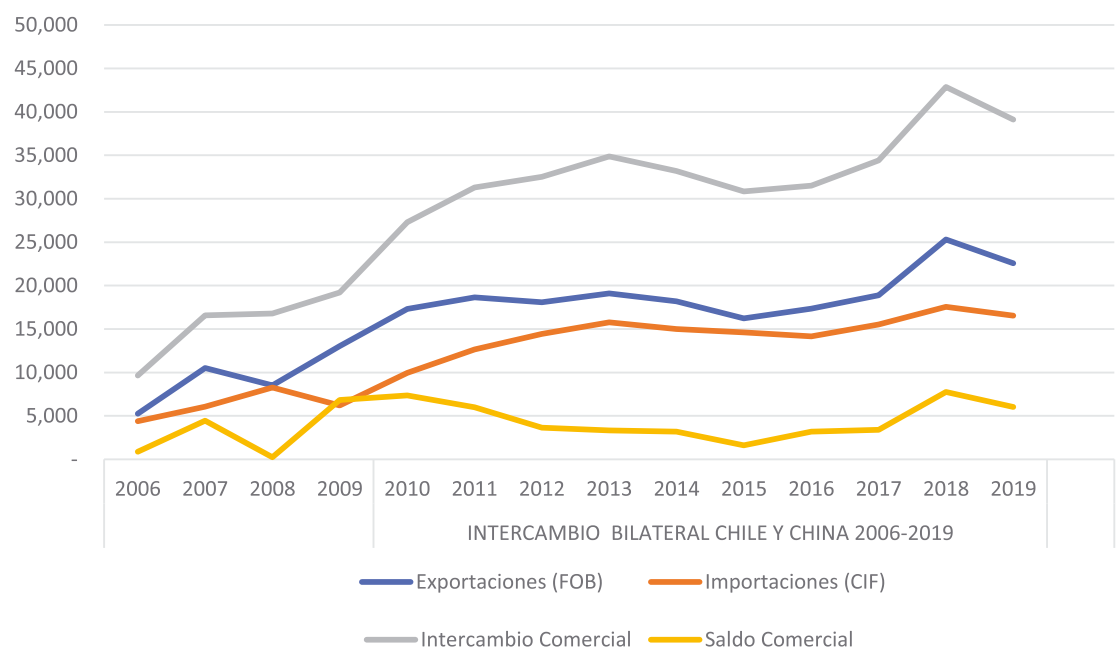

Gráfico No1: El intercambio comercial Chile-China 2006-2019.

Fuente: Elaboración propia según datos proporcionados por la DIRECON, Dpto de Estudios (2006-2013) y SUBREI, Dirección de estudios (2014-2019).

Los años de mayor intercambio comercial fueron el 2017 y 2018 con cifras de US\$34 408,1 millones y de US\$42 867,1 millones respectivamente. El año siguiente 2019 se vio afectado por la caída del precio del cobre implicando una grave crisis para la economía del país, producto de un menor retorno en divisas (SUBREI, 2019).

Es necesario recordar que en los años 90 e inicios del 2000, las relaciones comerciales chilenas con el Asia-Pacífico eran muy restringidas y en el caso de China se contaba con un personal muy reducido en la Embajada de Chile. Los escasos recursos económicos eran una restricción a la presencia de técnicos especializados, siendo esto solucionado años después, al asumir el agregado comercial y el agregado agrícola un fuerte protagonismo, contando con el apoyo permanente de PROCHILE. ${ }^{5}$ Las dificultades para entender la cultura, como también la lengua al momento de cualquier negociación, eran una visible limitante.

El embajador Fernando Schmidt, director de Asia-Pacífico de la Cancillería Chilena refiriéndose a ese contexto, en entrevista de estudio expresó:

5 PROCHILE, organismo dependiente de la Dirección General de Relaciones Económicas Internacionales, MINREL, responsable de promocionar, difundir y organizar ferias sectoriales dando a conocer los productos chilenos en el mercado mundial. Hoy este organismo forma parte de la SUBREI. 


\begin{abstract}
China está mirando a América Latina con ojos distintos, primero como suministrador, como fuente de materias primas. Para asegurarse esas fuentes de materias primas, sabe que necesita una determinada cantidad de inversiones (...) y mira a América Latina en el largo plazo como un contrapeso a los equilibrios mundiales (Schmidt, 2007).
\end{abstract}

En enero del 2005, en Beijing, fue el primer Summit y luego de cinco rondas, en octubre, se terminaron las negociaciones, quedando sujeto a la rectificación del Congreso Nacional. Los acuerdos fueron: aumentar el volumen de exportaciones e importaciones eliminando el cobro de aranceles, estableciendo un periodo de desgravamen a 10 años; en materia económica, fijar una normativa regulatoria para el comercio bilateral y favorecer la inversión extranjera; y en el ámbito político "buscó el reconocimiento de una cierta diferenciación de Chile en caso de turbulencias económicas internacionales; al tener un sistema de solución de controversias preciso (...) así el riesgo debiera disminuir” (Matus, 2006, p. 23).

La negociación se pactó en tres etapas:

I. Un Tratado de Libre Comercio en Bienes y se suscribió en Busán, Corea del Sur en el marco de la Cumbre de Líderes APEC en noviembre del 2005.

II. Un Acuerdo Suplementario de Comercio de Servicios, firmado en el marco de la visita a China, de la presidenta Bachelet en abril del 2008 y este entró en vigencia en el 2010.

III. Un Acuerdo Suplementario de Inversiones, dentro del Marco Cumbre de Líderes APEC-Vladivostok en septiembre 2012 y este entró en vigencia en febrero 2014 (SUBREI, 2021).

\title{
5 China, primer socio comercial y productos relevantes chilenos en las exportaciones
}

El TLC con Chile, como ya se dijo, fue el primero que negoció China con un país que estuviera fuera del bloque de ASEAN, marcando un punto de inflexión al buscar un país de América Latina como socio comercial. Esto nos permite hoy día poder hacer un análisis de los productos que han sido tradicionales en las exportaciones como también reconocer exportaciones recientes con productos que tienen un alto impacto en el consumo doméstico chino. Debemos considerar la diversidad de productos que a lo largo de estas cinco décadas han sido introducidos en el mercado chino, variando estos en valores y en volúmenes según períodos y las estaciones del año.

Serán analizados para este artículo los siguientes productos, ordenándose según las estadísticas de la Aduana de Chile según valores de exportación al año 2020, tomándose como años de referencia 2012-2020 (Aduanas, 2021). De acuerdo a lo señalado, los seis primeros productos en cuanto a valores expresados en 
millones de dólares son: el cobre, minerales de hierro y sus manufacturas, cerezas, celulosas, carne de porcino y vino. Es necesario decir que otras mercancías como la harina de pescado, los productos del mar con salmones y truchas entre otros y las uvas frescas fueron en su momento productos estrellas, perdiendo hoy los primeros lugares, como lo veremos a continuación por rubro.

Cabe señalar que se tomó la decisión para este artículo de trabajar los productos en base a sus valores de exportación en millones de dólares, lo cual se ve reflejado en el ingreso de divisas al país, aún cuando el volumen de las exportaciones expresado en toneladas o según correponda, no siempre coincide con un aumento o disminución del valor exportado. El espacio del texto no permitía una mayor extensión al respecto.

\subsection{El cobre, un producto ícono en las expotaciones chilenas}

El presidente de China Minmetals, ${ }^{6}$ Zhou Zhongshu, visitó Chile con motivo de la celebración de los 40 años de la firma de la relación diplomática el año 2010 y en el contexto del seminario organizado por la Universidad Andrés Bello, señaló:

Chile es el segundo mayor socio comercial de China en América Latina, mientras que China se ha convertido en el mayor socio comercial de Chile (...) en el campo de la minería, China es el mayor consumidor mundial de cobre, mientras que Chile es mundialmente conocido como el país del cobre, líder mundial en producción y exportación de este metal. Un 30\% aproximado de las importaciones de cobre que hace China proceden de Chile (Zhou Zhongshu, 2011, p. 23).

Dada la relevancia para la economía chilena de las expotaciones de cobre, el Gráfico N² nos permite observar las exportaciones de cobre en el período 20122020 con un valor de US\$10 275,5 millones y US \$7 788,4 millones respectivamente. Cabe decir que a la firma del TLC el año 2005, las exportaciones de cobre sumaban US\$3 396,8 millones según el Informe Comercio Exterior de Chile (DIRECON, 2006)

Es interesante señalar que los años anteriores al período 2012-2020, fueron los años de mayor bonanza ecónomica considerándose el 2010 con exportaciones de US\$ 11 055,6 millones y el 2011 con US\$ 11 576,1 millones permitiéndole al país un ingreso significativo con un precio promedio de la libra de cobre sobre los US\$ 3,59 en los años 2010-2014, muy superior al precio de los años siguientes (Economía y Negocios, 2017, p. 11).

6 Empresa líder China en la prospección, explotación, fundición y procesamiento de metales y otros productos de la minería. Fue fundada en 1950 y los vínculos con Chile se remontan a la década del 70, siendo un actor directo en la evolución del comercio chileno y la búsqueda de una proyección común. 


\section{Exportaciones de Chile a China-Cobre (US\$ FOB)}

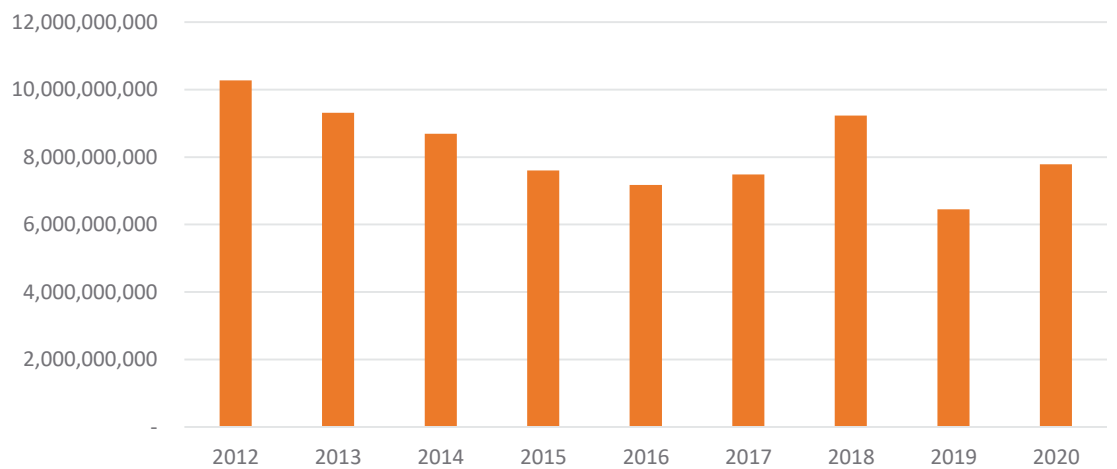

Gráfico N²: Exportaciones de Chile a China de cobre en período 2012-2020. Fuente: Elaboración propia según datos proporcionados por Aduanas de Chile, Comercio Exterior, 2021.

Chile, primer exportador mundial de cobre y monoproductor, por décadas ha mantenido la discusión sobre la forma de tener un valor agregado al producirlo y exportarlo. El expresidente Ricardo Lagos, en entrevista realizada, recordó una reunión con $\mathrm{CESCO}^{7}$ diciendo:

\footnotetext{
Chile mantiene el 32\% de la producción mundial, y el 30\% de las reservas, o sea 1/3 de los camiones están en Chile. Si a esos camiones le agrego una tuerca, ya estamos en un proceso de integración hacia atrás, lo que es necesario para seguir pensando sobre cómo debemos potenciar nuestra producción. Chile gasta US\$ 600 millones en importar camiones y los demás elementos. Esto ha variado. Primero fue de Escandinavia y posteriormente de Japón (...) Yo quiero que algunas de estas cosas, se produzcan acá. Pensemos en Perú, en Colombia. Ellos van a ser la gran sorpresa por sus propios recursos mineros, no saben cuántos son, ni tampoco las reservas futuras que tienen y Chile aquí tiene una oportunidad (Lagos, 2015).
}

La transacción del cobre en el mercado mundial ha cambiado el presente año, debido al aumento de la demanda de China, al reactivarse diferentes sectores de su economía luego de la pandemia. Esto ha producido un alza en sus precios en estos primeros meses de 2021, con un precio de 4,03 al 9 marzo, implicando mejores expectativas para el mercado interno al estar en estricta relación con el Presupuesto Anual de la Nación en Chile. Cabe decir que el febrero reciente el precio del cobre superó el nivel de 4,2 dólares la libra, aproximándose a sus máximos históricos marcados en febrero de 2011 (Dailyfx, 2021, febrero 25). Las proyecciones realizadas

7 Center of Cooper and Minning Studies. Referencia hecha por el expresidente Lagos al ser invitado en la reunión anual de abril 2015. 
por la Comisión Chilena del Cobre (COCHILCO) consideran un precio promedio de US\$3,3 la libra para este año (COCHILCO, 2021), lo que implicaría un aumento en los ingresos fiscales importantes en un período de recesión en medio de la crisis Covid19.

\subsection{El hierro, un mineral con altos niveles de competitividad}

El acceso a los mercados donde se produce y se exporta el hierro es clave al momento de la planificación de la actividad industrial de un país, dadas las demandas por acero, siendo esto determinante en la industria metalúrgica, automotriz e inmobiliria. A esto debemos sumar los proyectos de infraestrucura de una red vial que permita mejores accesos y conectividad. El mercado del hierro a nivel mundial está determinado casi en un 50\% por las demandas del mercado chino, siendo Australia el primer productor mundial. Dada la pandemia, algunos centros mineros se vieron afectados, sin embargo, en el caso de Australia, este mantuvo sus rangos de producción. La reducción de la producción en Brasil, segundo productor mundial, ha generado un nuevo ajuste en el precio aún cuando China, más allá de la pandemia, mantendrá sus demandas en forma creciente (Minning Press, 2021).

Las exportaciones chilenas de hierro a China tienen ya más de cuatro décadas, pero siempre se mantuvieron con volúmenes menores y muy fluctuantes. Sin embargo, por razones puntuales del mercado mundial y la pandemia el año 2020, al revisarse las estadísticas de Aduanas de Chile, aparece siendo el segundo producto en valor de exportaciones con una cifra en el año 2012 de US\$1 031,7 millones y el 2020 alcanzando US $\$ 1$ 436,7 millones como se puede apreciar en el Gráfico №3. Es necesario señalar que el año 2015 tuvo una baja significativa con US\$460,8 millones, representando un tercio del valor del año 2020.

Las variaciones en este producto son las más relevantes entre los productos señalados y requieren de un análisis mayor escapando a esta investigación, considerando variables como un escenario de pandemia, un contexto internacional marcado por los conflictos comerciales China-USA y las tendencias de los otros productores de hierro, siendo ellos en orden decreciente Brasil, India, China y Rusia.

\subsection{El impacto de las cerezas chilenas en el mercado chino}

El ingreso de las cerezas a China marcó un hito en el comercio exterior de Chile, ya que al momento de producirse los primeros embarques no era predecible saber el valor nominal y sentimental futuro de este producto. Su llegada para las fiestas del 


\section{Exportaciones de Chile a China- Minerales de hierro y sus concentrados US\$ FOB}

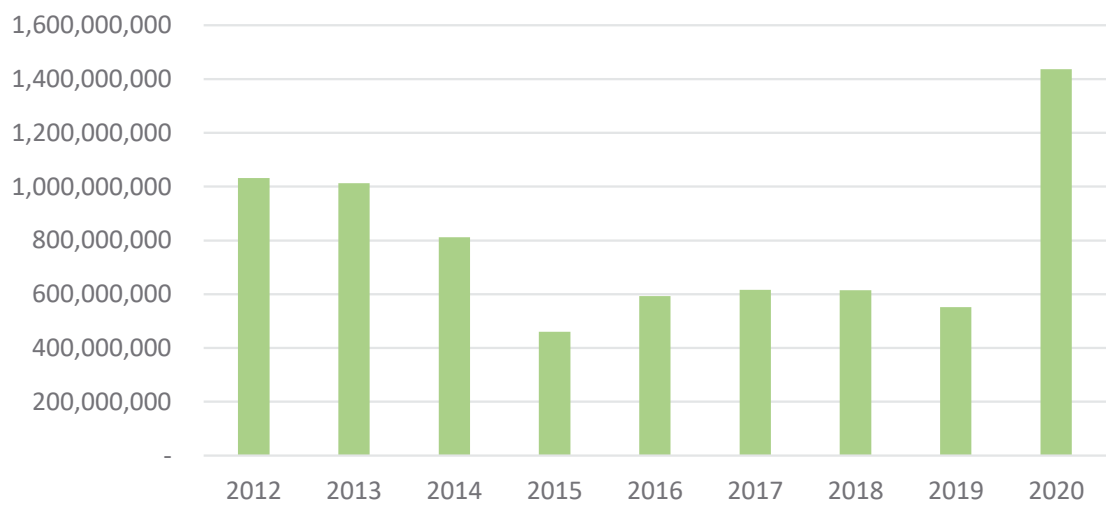

Gráfico N`3: Exportaciones de Chile a China de hierro en período 2012-2020. Fuente: Elaboración propia según datos proporcionados por Aduanas de Chile, Comercio Exterior, 2021.

Año Nuevo Chino impactó dadas sus propiedades de sabor, color rojo emblemático y forma de corazón de esta fruta chilena. Su distribución y la posibilidad de regalarlas como un signo de afecto en una caja con un diseño exclusivo, representan valores culturales y un signo de amistad al ocurrir el Año Nuevo Lunar. Es

\section{Exportaciones de Chile a China-Cerezas

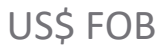

$1,600,000,000$

$1,400,000,000$

$1,200,000,000$

$1,000,000,000$

$800,000,000$

$600,000,000$

$400,000,000$

$200,000,000$

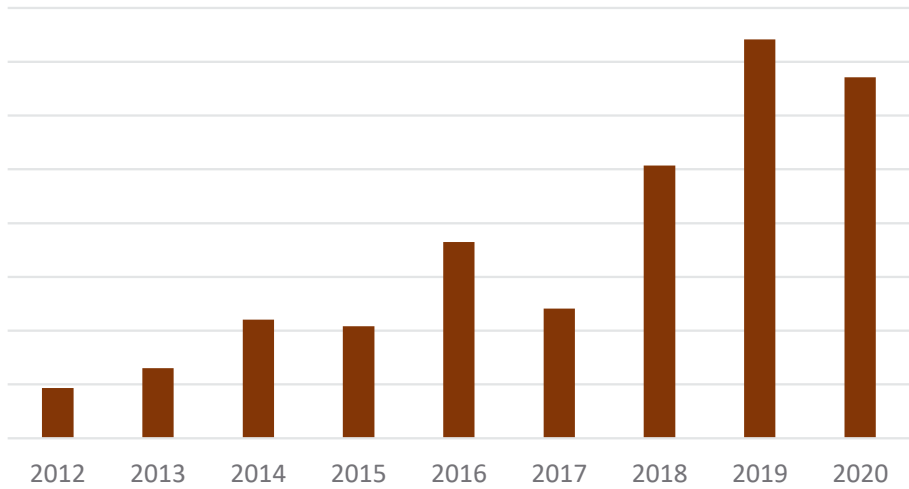

Gráfico N 4 : Exportaciones de Chile a China de cerezas en período 2012-2020. Fuente: Elaboración propia según datos proporcionados por Aduanas de Chile, Comercio Exterior, 2021. 
interesante señalar que el primer embarque de cerezas ingresó el año 2012 con un valor de US\$186,3 millones para llegar solo en nueve años a una cifra de US\$1 342,8 millones el 2020, siendo el tercer producto de mayor importancia en las exportaciones (Gráfico $\mathrm{N}^{\circ} 4$ ).

Su promoción fue una estrategia de marketing desde Prochile y la Asociación de Exportadores de Chile (ASOEX). Para los chinos es un producto emblemático y, según noticia publicada, les llaman la atención los colores rojo y verde que representan buena suerte y riqueza (EMOL, 2017). La podríamos considerar el sello rojo de la amistad Chile-China.

\subsection{Las exportaciones de celulosa, y su tendencia desde la firma de relaciones diplomáticas}

Estas se iniciaron en los años 70 y han variado en volumen según las décadas. En este caso está considerada solo la celulosa, sin desconocer otras partidas como es la pasta química y la madera. En las primeras décadas de relaciones diplomáticas entre China-Chile este rubro ocupó siempre el segundo lugar, salvo algunos años puntuales. Sin embargo, al observarlo hoy, se localiza en un cuarto lugar.Las rebajas arancelarias producto de la firma del TLC permitieron un aumento en los volúmenes de exportación y, dado el crecimiento de China en las últimas dos décadas, las necesidades por fabricar papel, cartón, y otros productos afines aumentaron, como también los rollizos de madera para en el sector inmobiliario, considerando el masivo proceso de urbanización en el interior del país.

Las cifras de exportación para este rubro al año 2012 era de un valor de US\$898,8 millones y luego, en el 2020 fue de US\$1 293,4 como se expresa en el Gráfico $\mathrm{N}^{\circ} 5$. Cabe recordar que al momento de la entrada en vigencia del TLC el 2006, las exportaciones en valor correspondían a US\$331,1 millones.

Es interesante observar el alza en este rubro que se produce el año 2018 con un valor de US\$2 003,7 millones, originando un significativo ingreso de divisas a Chile, aún cuando hay que tener presente que esto varía también por la disponibilidad del producto y fluctuaciones en el mercado mundial.

\subsection{La carne de porcino chilena, una oferta reciente para las demandas alimenticias chinas}

La dieta alimenticia China siempre ha tenido como un producto fundamental la carne de porcino, siendo esto fundamental en el consumo doméstico y diario. Cabe recordar 


\section{Exportaciones de Chile a China- Celulosa \\ US\$ FOB}

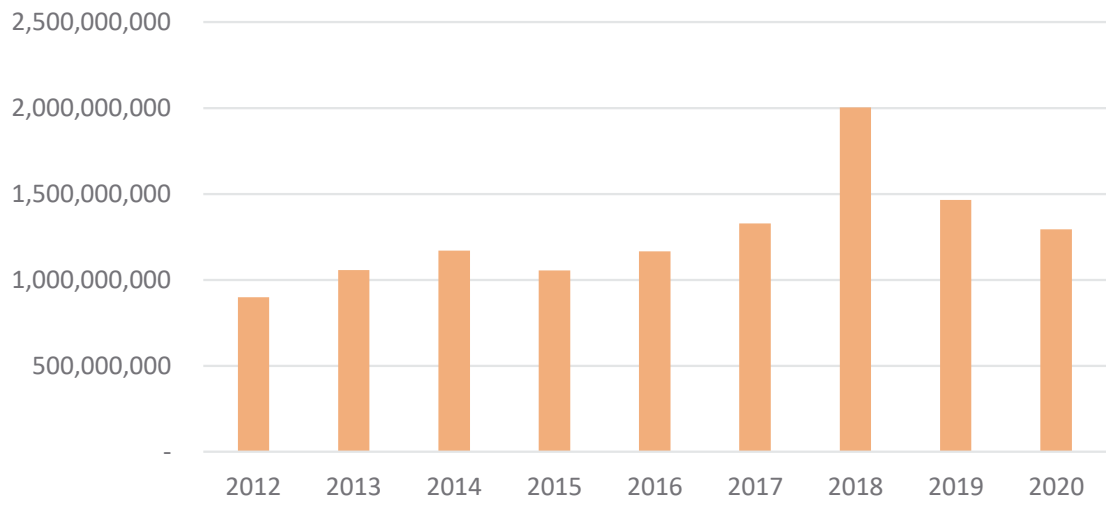

Gráfico N5: Exportaciones de Chile a China de celulosa en período 2012-2020. Fuente: Elaboración propia según datos proporcionados por Aduanas de Chile, Comercio Exterior, 2021.

Exportaciones de Chile a China -Carne de porcino.

US \$ FOB

$600,000,000$

$500,000,000$

$400,000,000$

$300,000,000$

$200,000,000$

$100,000,000$

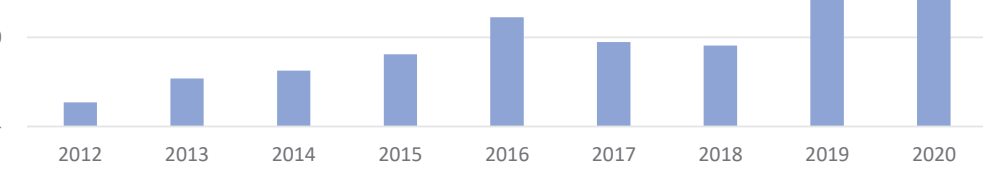

Gráfico N6: Exportaciones de Chile a China de carne de porcino en período 2012-2020. Fuente: Elaboración propia según datos proporcionados por Aduanas de Chile, Comercio Exterior, 2021. 
que, recién iniciadas las relaciones diplomáticas en los años 70, un producto emblemático que llegaba a Chile en lata, era el luncheon meat o “carne de cerdo”, siendo esto producto de acuerdos comerciales incipientes que se firmaron en esa década.

Las cifras de exportación para este rubro al año 2012 era un valor de US\$27,04 millones y luego, en el 2020 fue de US\$556,3 millones como se expresa en el gráfico siguiente correspondiendo a un aumento en forma sorprendente de un 1,956\% (Gráfico $\left.\mathrm{N}^{\circ} 6\right)$.

\subsection{El consumo del vino, un giro en las costumbres y las comidas de los chinos}

Las primeras exportaciones de vino, uvas frescas y mostos fueron, hacia 1985, con las viñas San Pedro y Viña Montes. El mercado era muy restringido y enfocado a extranjeros y diplomáticos (Murray, 2007). Años después el consumo se expandío al haber un aumento significativo del poder adquisitivo, produciéndose un giro en las costumbres y en la dieta alimenticia de los chinos. Así las exportaciones de vino al 2012, eran un valor de US\$82,03 millones y luego el 2020 fue de US\$185,6 millones como se ve en el gráfico siguiente (Gráfico $\mathrm{N}^{\circ} 7$ ).

Este crecimiento en la demanda por los vinos chilenos significó ya no solo su comercialización en las zonas costeras como Hong-Kong, Shanghai y Beijing, sino

\section{Exportaciones de Chile a China - Vino - US \$ FOB}

\section{$300,000,000$}

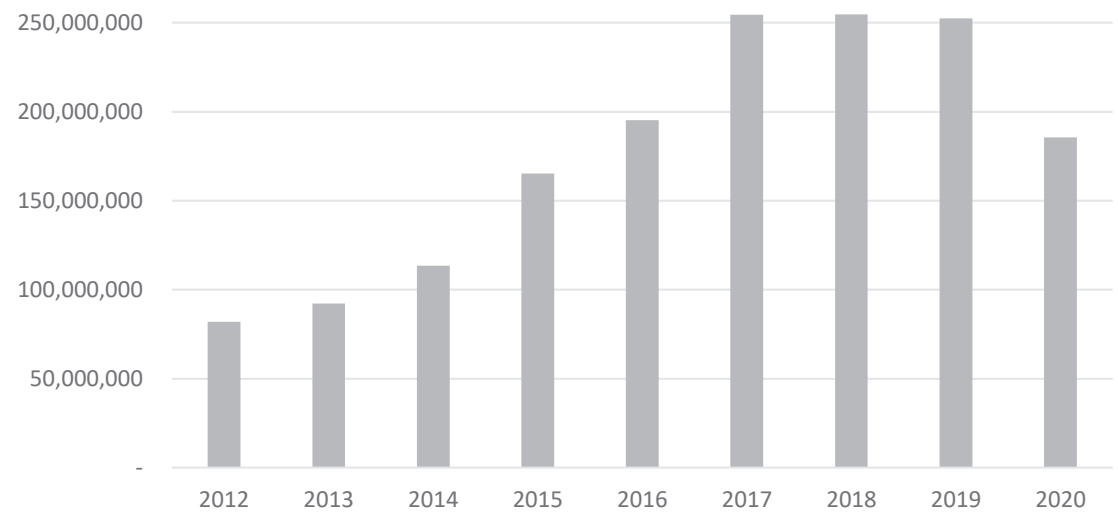

Gráfico N7: Exportaciones de Chile a China de vino en período 2012-2020. Fuente: Elaboración propia según datos proporcionados por Aduanas de Chile, Comercio Exterior, 2021. 
también abrir mercados en las provincias del interior, implicando un compromiso de los empresarios chilenos en el mercado asiático, y entender los códigos culturales de su contraparte. Junto a esto, es posible recordar la llegada de fruta fresca chilena y uvas al firmarse un Convenio Fitosanitario en 1998, permitiendo cumplir con la normativa al llegar los embarques con la tecnología adecuada y cámaras de frío con atmósfera modificada.

Al hacerse una retrospectiva en el ámbito económico y comercial del 2009, China pasó a ser el primer socio comercial de Chile, desarrollándose una nueva estrategia. Esto quedó de manifiesto con las visitas realizadas a China por la Presidenta Bachelet en abril 2008 ampliando el TLC al sector de servicios (MINREL, 2014). A su vez, el Primer Ministro Wen Jiabao visitó Chile en junio de 2012, incorporando un nuevo capítulo del TLC en materia de inversiones.

El Premier Li Keqiang en mayo de 2015 estuvo en Chile, en CEPAL, donde anunció proyectos de infraestructuras en la línea de Iniciativa de la Franja y la Ruta y la creación del Banco de Inversiones en Infraestructura Asiático. Al respecto señaló:

\footnotetext{
Mi gira es como una visita a viejos amigos, a quienes no he visto hace tiempo, me hace sentir como volver a casa (...) en mi intervención en la Cumbre Empresarial China-Brasil dije que esta región constituye la piedra angular de la paz y la estabilidad mundiales, una fuerza emergente de la prosperidad y crecimiento globales, y un destacado representante de la convivencia inclusiva y armoniosa de la humanidad (Li Keqiang, 2015).
}

La visita de estado del Presidente Xi Jinping en noviembre 2016, confirmó dichos anteriores:

Hace cinco años, hice una visita a Chile que me dejó gratas impresiones. Hoy, volviendo a pisar esta hermosa tierra, percibo un doble cariño abrigando infinitas expectativas. Situados China y Chile en las antípodas de la Tierra y separados por una gran distancia geográfica, ambos pueblos han mantenido un intercambio y un lazo indisoluble que se remontan a tiempos lejanos... China y Chile no solo llevan nombres de hermanos, a los dos pueblos los une una amistad fraternal (Xi Jinping, 2016).

\section{Las bases construidas desde lo cultural fortalecieron los lazos póliticos-económicos}

Las relaciones entre Chile y China son el producto de cinco décadas de sólida amistad. El tema de la cercanía ideológica construida desde la periferia del tercer mundo, junto al liderazgo de Salvador Allende, Pablo Neruda y José Venturelli, permitieron en los años 50 un espacio privilegiado frente a otros países de América Latina. La fundación del ICCC fue la herramienta clave, siendo el impusor de los 
viajeros a la República Popular China. Así, las invitaciones para conocer la Nueva China se extendían a intelectuales, artistas, líderes políticos y dirigentes sindicales, como también a representantes del Estado dentro de un contexto de guerra fría global (Ahumada, 2020).

El rol de José Venturelli, y sus vínculos estrechos con las autoridades chinas, sumado a su amistad con el expresidente de Chile Salvador Allende, permitió el "gesto emblemático" de la firma de relaciones diplomáticas como una respuesta de reciprocidad frente al país asiático. Dada la acción de una diplomacia cultural que se extendió hacia toda la región de América Latina, un grupo significativo de viajeros visibilizaron el modelo maoísta donde lo cultural siempre se sostuvo como un pilar fundamental para el desarrollo de la RPCH y el legado del artista chileno permaneció en las nuevas generaciones de China.

Producido el golpe militar en 1973, las relaciones diplomáticas no se interrumpieron, sustentándose la política exterior China en la no interferencia en asuntos internos de otro Estado, como parte de los Cinco Principios de Coexistencia Pacífica (Malena, 2010, p. 33). Esto implicó que durante la dictadura de Pinochet, dos regímenes de ideas antagónicas convergieran en acercamientos mutuos, facilitando incipientes contactos comerciales, considerando que China comenzaba su proceso de modernización con Deng Xiaoping. La cultura y los rasgos propios de la civilización China se mantendrían como un eje medular.

El año 2016, fue consagrado por CELAC como el Año de la Cultura ChinaAmérica Latina, implicando la invitación de artistas consagrados latinoamericanos. En ese contexto, la Academia China de Bellas Artes (CAFA), invitó a exponer la obra del artista chileno, Masters of Latin Américan: José Venturelli, siendo exhibida por más de tres meses itinerando por las ciudades de Beijing, Nanjing y Shanghai. Todo esto permitió una vez más entrelazar las relaciones China-Chile, teniendo presente que lo político y economíco solo se construye cuando la cultura forma parte del legado patrimonial. El propio Venturelli decía que su arte como muralista lo impulsaba a los espacios públicos y a generar una conciencia crítica. Este año 2021, nuevamente su obra está presente en el Centro Cultural Gabriela Mistral (GAM) José Venturelli y China. 50 años de los vínculos diplomáticos de Chile y China. El aporte intercultural de José Venturelli. Así, los lazos entre Chile y China se sustentan en una base con raíces lejanas y profundas, poco conocida por los propios chilenos.

Transcurridos 15 años de la firma del TLC, hay importantes logros en un contexto intercultural, haciendo mención al Memorándum de Entendimiento para la enseñanza del chino (2004) en un Plan Piloto con el Ministerio de Educación de Chile. Esto permitió que el Instituto Confucio (Hanban) mandara profesores chinos para certificar el nivel de la lengua al rendir las pruebas HSK y el Young Chinese Test (YCT). A esto podemos agregar universidades que han ido incorporando programas para enseñar la lengua y la cultura de China. Cabe destacar la Universidad Católica de 
Chile y la Universidad Santo Tomás, con los Institutos Confucio, junto a la Universidad Andrés Bello, la Universidad Central, la Universidad de Santiago y la Universidad del Desarrollo, que cuentan con programas específicos, con diferentes intercambios de estudiantes y académicos, como también con profesores chinos como invitados especiales.

\section{Acuerdos recientes en el ámbito tecnológico y científico que marcan un hito}

El 2015 visitó nuestro país el Dr. Zhang Yaping, zoólogo y vicepresidente de la Academia China de Ciencias (CAS), buscando firmar acuerdos de cooperación reconociendo el potencial de Chile en energías renovables. En su ponencia señaló:

\footnotetext{
El gasto total en investigación es de US\$ 191000 millones, correspondiendo a más del 2\% del PIB del país, donde existen programas completos de laboratorios para estudiar microciencia (...) China es el número 1 en investigación a nivel mundial y de todos los cientistas más renombrados 152 son de China (...) A los avances anteriores, agregó los logros en energías renovables, como también las grandes oportunidades que tiene Chile en el ámbito de la astronomía, la hidrofísica, la innovación conjunta en minería como también en materia de sismología donde Chile puede aportar mucho por su experiencia (Zhang, 2015).
}

Los acuerdos al respecto se han ido sumando, pudiendo ver importantes avances en materia de proyectos de inversión vinculados a la producción de energías renovables e inversiones en materia eléctrica, donde la empresa China SGID compró la empresa eléctrica CGE, disputando el liderazgo en el sector eléctrico chileno, como ya se dijo. Otros proyectos en el ámbito de la astronomía se suman, donde la instalación de obsevatorios va a la vanguardia de la investigación científica, siendo Chile un país con significativas ventajas comparativas al tener cielos muy limpios y características atmosféricas únicas a nivel mundial.

En materia tecnológica, poder asegurar una red digital $5 \mathrm{G}$ es uno los requerimientos del sector industrial y productivo chileno. Los acuerdos recientes firmados con la empresa Huawei y la visita del Presidente Piñera el 2019 a la ciudad de Shenzhen, centro tecnológico del gigante asiático, de alguna manera facilitarán nuevas tecnologías acordes con el impacto de la inteligencia artificial (Cooperativa, 2019).

Sin embargo, lo más relevante fue el memorándum de Entendimiento firmado el año pasado, con motivo del Covid-19, entre el laboratorio chino Sinovac Biotech Ltda. y el rector de la Universidad Católica de Chile, Ignacio Sánchez, estableciendo un intercambio de conocimientos e información para realizar ensayos clínicos en Chile a través de la prueba de la vacuna en personas. Así, la población chilena tendría acceso a una vacunación masiva llegado su momento $\mathrm{y}$ 
asegurando los requerimientos para la inoculación contando con los insumos necesarios. Cabe decir que este laboratorio ya había tenido experiencias anteriores con la vacuna de la influenza y de la hepatitis en Chile. Al respecto, el rector señaló:

Esta pandemia nos ha recordado que no somos seres ni países aislados, necesitamos de la colaboración para enfrentar de manera más eficiente aquellos desafíos que requieren de soluciones que puedan alcanzar toda la población. Esta colaboración es una luz de esperanza en el difícil momento que estamos viviendo (Sánchez, 2020).

Transcurrido ya casi un año de la firma de este acuerdo y cumplidas las etapas requeridas por el proyecto, Chile sigue siendo un país con altos niveles de contagio según las estadísticas diarias que emite el Ministerio de Salud. Sin embargo, a esta fecha Chile se convierte en uno de los líderes mundiales en proceso de vacunación con 1,08 dosis diarias por cada 100 mil habitantes (Deutsche Welle, 2021). Esto implica a la fecha que ya hay 4618528 personas vacunadas según registros de Minsal al 11 de marzo 2021.

El hecho de que Chile haya podido generar una logística sanitaria inmediata se remonta a la década del 50, como lo señaló el Ministro de Salud, Enrique Paris al decir: "esto no es obra de un gobierno, es producto del esfuerzo que ha hecho el servicio público cuando se unió la opinión de los médicos Eduardo Cruz-Coke y Salvador Allende y juntos crearon el Sistema Nacional de Salud. Esa es nuestra fortaleza” (El Mercurio, 2021, Cuerpo C p. 1). De alguna manera, el servicio de salud público chileno respondía a un nuevo desafío no siendo una acción reciente, sino los programas implementados décadas anteriores, más allá de las diferencias políticas enormes que lograron cimentar las bases de un sistema sanitario fuerte y sólido fundamentado en la Atención Primaria de Salud (Crónica Digital, 2021). La pandemia daba inicio a una nueva etapa estratégica de colaboración mutua entre ambos Estados en materia de salud pública.

\section{Consideraciones finales}

¿Qué podemos preguntarnos luego de un largo recorrido de 50 años?

La respuesta debemos plantearla en función de un futuro posible en las próximas décadas y es allí donde debemos poner las energías y fortalezas. La ruta se construyó con anterioridad al llamado realizado por el presidente Xi Jinping, al llamar a Chile junto a los otros países de América Latina y el mundo a ser parte del sueño común expresado en One Belt, One Road. Esto identificando a una sociedad China con un mayor poder adquisitivo que busca nuevos patrones de conducta, pero sin perder de vista los pilares de sacar a la población China de la pobreza y lograr una asistencia coordinada donde las regiones costeras de mayores recursos 
apoyan a las zonas del interior con menor acceso. Bien sabemos cómo la conectividad es la clave y sin acceso no hay desarrollo posible, como ha quedado demostrado en la actual pandemia a nivel mundial, y China cuenta con tecnología $5 \mathrm{G}$ y puede implentar una ruta digital.

A su vez desde Chile, ¿qué podemos preguntarnos?

Una ruta no es un camino ya trazado, es una posibilidad de abrirse a nuevos desafíos y oportunidades donde las condiciones actuales de Chile, con conflictos sociales importantes por la falta de equidad, nos invitan repensar lo ya construido en décadas anteriores, como fue descrito en el artículo. Este recorrido está marcado por la confianza mutua y es en este espacio donde se abre una nueva etapa, donde ya no solo lo cultural o lo comercial son lo primordial, sino que también a ello se suman los espacios de cooperación para proyectos conjuntos en materia de energías renovables, estudios compartidos en materia sismológica, implementación de observatorios astronómicos y la creación de ciudades sustentables con transporte eléctrico, entre otros. Sin embargo, avanzar en materia de investigación científica como ha sido el trabajo conjunto con la vacuna Sinovac y la implementación de laboratorios para su posible fabricación son ya uno de los pasos que se comienzan a dar. La sinergia común construida podrá ser una nueva apuesta que no solo involucre a Chile, sino también a otros espacios extraregionales y a Latinoamérica.

Los próximos años postpandemia serán el momento para impulsar políticas de Estado que favorezcan a cada país y que se enmarquen en la línea de la Agenda 2030 de la ONU y la búsqueda de un desarrollo sostenible. Es en esta línea en que Chile deberá sumarse a nuevos proyectos, en una región marcada por la pobreza extrema y la falta de acceso a la educación. El camino recorrido junto a China podrá impulsar así un rol en organismos multilaterales.

\section{Referencias bibliográficas}

Aduanas, de C. (2021). Exportaciones por continente y país. Comercio Exterior. Aduanas de Chile, Santiago: Servicio Nacional de Aduanas. https://www.aduana.cl/exportaciones-porcontinente-y-pais/aduana/2018-12-13/115605.html.

Ahumada, M. (2020). Viajeros a la República Popular China: José Venturelli, los intelectuales, políticos y parlamentarios chilenos en los años cincuenta y sesenta. TRANSMODERNITY: Journal of Peripheral Cultural Production of the Luso-Hispanic World, 9(3). Retrieved from https://escholarship.org/uc/item/7j81d158.

Banco Mundial. (2021a). Datos Estadísticos. Banco Mundial. https://datos.bancomundial.org/ indicator/SP.POP.TOTL?locations=CL.

Banco Mundial. (2021b). Datos Estadísticos. Banco Mundial. https://datos.bancomundial.org/ indicador/NY.GDP.MKTP.KD.ZG. 
Banco Mundial. (2021c). Datos Estadísticos. Banco Mundial. https://datos.bancomundial.org/ indicator/SP.POP.TOTL.

Comisión Chilena del Cobre (COCHILCO). (2021). Cochilco eleva las proyecciones del precio del cobre para el 2021 a 3.3 la libra. Santiago: Ministerio de Minería. https://www.cochilco.cl/ Paginas/Inicio.aspx.

Cooperativa. (2019). Piñera llega a Shenzhen, el Silicon Valley de China. Radio Cooperativa. https://www.cooperativa.cl/noticias/pais/relaciones-exteriores/china/pinera-llega-ashenzhen-el-silicon-valley-de-china/2019-04-27/080554.html.

Crónica Digital. (2021). Ministro Paris reconoce a Allende como uno de los creadores del sistema público de salud. https://www.cronicadigital.cl/2021/02/12/vacunacion-ministro-desalud-reconoce-a-allende-como-uno-de-los-creadores-del-sistema-publico-de-salud/.

Dailyfx. (2021). El precio del cobre se dispara en un $23 \%$ en febrero y mira a máximos una década después. DilyFx. https://www.dailyfx.com/espanol/analisis_del_mercado/cotizacion_ materias_primas/2021/02/25/El-precio-del-cobre-se-dispara-un-23-en-febrero-y-mira-amaximos-una-decada-despues.html.

Deutsche Welle. (2021). Chile se convierte en líder mundial en administración de la vacuna contra Covid-19. Deutsche Welle. https://www.dw.com/es/chile-se-convierte-en-l\%C3\%ADdermundial-en-administraci\%C3\%B3n-de-la-vacuna-contra-covid-19/a-56820540.

Dirección General de Relaciones Económicas Internacionales (DIRECON). (2006). Comercio Exterior de Chile. Cuarto Trimestre 2005. Ministerio de Relaciones Exteriores. http://www. sice.oas.org/ctyindex/CHL/DIRECON2005_s.pdf.

Economía y Negocios. (2017). Tres décadas de Chile en Cifras. Edición Especial. El Mercurio.

El Mercurio. (2021). Chile lidera en velocidad de vacunación en el mundo, pese a dsiparar avance en regiones. El Mercurio.

El Pulso. (2020). China SGID comprará CGE y entra a disputar liderazgo en el sector eléctrico chileno. El Pulso. https://www.latercera.com/pulso/noticia/china-sgid-comprara-cge-yentra-a-disputar-liderazgo-en-el-sector-electrico-chileno/ 6BDGM5GVEBHT3C74PYW3MBADPY/.

EMOL. (2017). Cerezas chilenas: ¿Cuáles son los precios que pagan los chinos y que causaron la queja asiática? EMOL. Diario electrónico. http://www.emol.com/noticias/Economia/2017/ 05/10/857684/Cerezas-chilenas-en-China.html.

Jiang, S. (2006). Relaciones bilaterales chino-chilenas en el umbral de una nueva etapa de desarrollo. In Chile y China: reflexiones para una agenda integral. Beijing: Instituto de Relaciones Internacionales Contemporáneas de China. Embajada de Chile.

Lagos, R. (2004). Discurso del Expresidente de Chile en la Cena de Honor con motivo de la Cumbre APEC en Santiago de Chile el 18 de noviembre de 2004. http://asiapacifico.ben.cL/china/ documentos/discursos.

Lagos, R. (2007). Entrevista de estudio realizada a Ricardo Lagos, Expresidente de Chile e impulsor de la firma de un TLC Chile-China, en Santiago de Chile, el 27 de diciembre 2007.

Lagos, R. (2015). Entrevista de estudio realizada a Ricardo Lagos, Expresidente de Chile en contexto de los 10 años de la firma del TLC, en Santiago de Chile, 23 de septiembre 2015.

Li, K. (2015). Discurso del premier chino en la sede de la CEPAL en Chile el 25 de mayo de 2015. http://spanish.people.com.cn/n/2015/0529/c31617-8899298.html.

Malena, J. (2010). China, la construcción de un país gigante. Buenos Aires: Céfiro.

Mancilla, L. (2003). Hoy es Todavía. José Venturelli, una biografía. Santiago: LOM. 
Matus, M. (2006). La política comercial de Chile. In Chile y China: reflexiones para una agenda integral. Instituto de Relaciones Internacionales Contemporáneas de China. Embajada de Chile.

Ministerio de Relaciones Exteriores (MINREL). (1970). Memoria Anual 1970. Ministerio de Relaciones Exteriores de Chile.

Ministerio de Relaciones Exteriores (MINREL). (2014). Canciller participa en visita a China junto a presidenta Michelle Bachelet. http://www.minrel.gov.cl/canciller-participa-en-visita-achina-junto-a-presidenta-michelle-bachelet/minrel/2014-11-13/181933.html.

Minning Press. (2021). El hierro de Australia revive tras la pandemia. Minning Press. http:// miningpress.com/nota/330865/el-hierro-de-australia-revive-tras-la-pandemia.

Murray, D. (2007). Entrevista de estudio al presidente de Viña Montes, realizada el 5 de enero 2008.

Neruda, P. (1992). Confieso que he vivido. Memorias. Buenos Aires: Ed. Planeta.

Schmidt, F. (2007). Entrevista de estudio al exdirector Asia-Pacífico, realizada el 18 octubre 2007. Spanish.xinhuanet. (2017). Xi Jinping elegido Secretario General del Comité Central del Partido Comunista Chino. Xinhuanet. http://spanish.xinhuanet.com/temas/201710CNPCCh/index. htm.

Subsecretaría de Relaciones Económicas Internacionales (SUBREI). (2019). China Anual. Ficha País Chile-China. Indicadores Macroeconómicos. Ministerio de Relaciones Exteriores. https://www.subrei.gob.cl/docs/default-source/default-document-library/chinaanual2019.pdf?sfvrsn=520a1b1_0.

Subsecretaría de Relaciones Económicas Internacionales (SUBREI). (2021). Chile-China. Acuerdo de Libre Comercio. Ministerio de Relaciones Exteriores. https://www.subrei.gob.cl/ acuerdos-comerciales/acuerdos-comerciales-vigentes/china\#: :text=El\%20Tratado\%20de \%20Libre\%20Comercio,Chile\%2C\%20en\%20noviembre\%20de\%20.

Vera Castillo, J. (1987). La política exterior chilena durante el gobierno de Salvador Allende 19701973. Santiago: Ediciones Instituto de Estudios de Relaciones Internacionales Contemporáneas (IERIC).

Wu H. (2011). Las relaciones sinolatinoaméricanas. In Y. T. Lee, \& H. Wu (Eds.), Chile y China. Cuarenta años de política exterior. Una trayectoria de Continuidad y perseverancia. RILEditores, CICIRy UDD.

Xi, J. (2016). Crear juntos un porvenir más espléndido para las relaciones entre China y Chile. El Mercurio. http://cl.china-embassy.org/esp/zldt/t1417908.htm.

Zhang, Y. (2015). Diálogos con la Ciencia: Nanotecnología. Santiago, Chile: Ex Congreso Nacional. Zhongshu, Z. (2011). El potencial de la cooperación entre Chile y China es enorme y su horizonte se amplía cada día. In F. Reyes Matta (Ed.), Chile y China 40 años: ¿Qué nos trae el futuro? Santiago: Universidad Andrés Bello, Centro de Estudios Latinoamericanos sobre China-CELC. 